schließlich auf das Begehren, vor dem Gesetz zu reden, statt die Bedingungen zu analysieren, unter denen ein solches ausnutzbares Begehren erzeugt wird. Und außerdem: paßt der Begriff »Menschenrechtsverletzung « überhaupt noch, wenn der Mensch, die Verletzung und der Verletzer ihre Eindeutigkeit verloren haben?

Wenn komplexe Beziehungen mit wechselnden Machtverhältnissen sich in der binären Strukturierung des Erfahrungsgehaltes der Menschenrechte nicht mehr erfassen lassen, tritt eine paradoxe Situation ein. Man kann dann - vor allem in westlichen Ländern - durchaus von der Einhaltung der Menschenrechte sprechen, und zwar selbst dort, wo Unrecht geschieht. Anders ausgedrückt: die Beachtung der Menschenrechte ist keine Garantie dafür, daß keine Gewalt im Recht stattfindet. Andere Formen der Gewaltausübung unterlaufen den Maßstab der Menschenrechte einfach. Wenn aber die Einhaltung der Menschenrechte zum Kriterium für die Zulassung in internationalen Zusammenschlüssen, letztlich zum Garanten für einen bestimmten wirtschaftlichen Standard der Länder wird, sind sie eine unmittelbar politische Angelegenheit von Staaten. Ihre Anerkennung verbürgt dann keine rhetorische Praxis. Sie sind ein wirtschaftlicher Faktor im Kalkül staatlicher Außenpolitik und auch eine bequeme Legitimationsfigur für internationales Handeln. Darin deutet sich die Transformation der Menschenrechte an: aus der Berufung auf Humanität ist ein Dispositiv geworden, um weltpolitische Vorgänge in Form von rechtlichen Akten zu fassen. In rechtlichen, menschenrechtlichen Begriffen zumal, wird eine Interessenpolitik westlicher Länder schließlich konsensfähig.

Mit einer Reformulierung der Menschenrechte gemäß ihrer Idee läßt diese Entwicklung sich nicht zurückdrängen. Das Sprechen im Namen der Menschenrechte ist unwiderruflich ein rechtliches des Staates geworden; der Diskurs der Menschenrechte ist kodifiziert und als solcher Gegenstand von Analyse und Kommentar wie andere Gesetzeswerke auch.

\title{
Hauke Brunkhorst \\ Sind Menschenrechte Aporien? \\ Kritische Bemerkungen zu einer These Hannah Arendts
}

$I$

An der Schwelle der modernen Verfassungsrevolutionen im I 8. Jahrhundert schien es, so schreibt Hannah Arendt in The Origins of Totalitarism, »fast selbstverständlich, daß diese beiden Dinge: Volkssouveränität und Menschenrechte, einander bedingen und sich gegenseitig garantieren."

Eine wechselseitige Garantie von Volkssouveränität und Menschenrechten ist aber so lautet Arendts zentrale These- unmöglich. Entweder der demokratische Wille des Volkes ist der "sterbliche Gott" des Thomas Hobbes, der niemandem Rechenschaft schuldet und an keine universelle Rationalität gebunden ist, oder es herrscht das

1 H. Arendt, Elemente und Ursprunge totalitarer Herrschaft, Munchen 1986, S. 454. Exemplarisch ist hier-
wie Ingeborg Maus, Zur Aufklarung der Demokratietheorie, Frankfurt 1992, gezeigt hat - die Position von Kants Rechtsphilosophie. 
Gesetz, das seine Legitimität allein der höheren Wahrheit der Menschenrechte verdankt. ${ }^{2}$ Die Menschenrechte lassen sich durch keinen Volkswillen darstellen. Sie stehen im demokratischen Diskurs nicht zur Debatte. Umgekehrt aber ist der Wille des demokratischen Souveräns einer Universalisierung im Lichte der Menschenrechte weder fähig noch bedürftig. Der souveräne Wille beugt sich keinem Gesetz, ist er doch die nicbt mebr zur Debatte stehende Quelle aller gesetzlichen Legitimität. Entweder die Herrschaft des Gesetzes verletzt und beleidigt die schrankenlose Freiheit des Willens, der sich selbst bestimmen will, oder die freie Willkür kommt unverkürzt zum Zuge und bricht das Recht. ${ }^{3}$

In jeweils letzter Instanz stehen weder die Menschenrechte, die sich auf die stumme Wahrheit der Natur stützen, noch der Wille des Volkes, der dem einsamen Entschluß eines jeden entspringt, zur Diskussion. Wenn wir diese Prämisse, die Arendt mit Carl Schmitt teilt, in Frage stellen, löst sich die Aporie der Menschenrechte und es eröffnet sich die Perspektive einer Kritik sowohl des klassischen Liberalismus mit seiner These vom Vorrang der Rechte wie des klassischen Republikanismus, der vom Vorrang des kollektiven Willens ausgeht.

In Arendts Aporie der Menschenrechte spiegelt sich noch einmal der Streit der deutschen Staatsrechtslehre um die Verfassung von Weimar. Souverän, so die These Hans Kelsens, ist allein das willenlose Gesetz, die anonyme Rechtsordnung. ${ }^{4}$ Souverän, so Carl Schmitts Antithese, ist allein der nicht rationalisierbare Wille des Volkes bzw. seines Führers. Voluntas oder ratio, - tertium non datur. ${ }^{5}$

Die Menschenrechte sind abstrakt und negativ, bloße Abwehrrechte gegen den Staat, die demokratische Freiheit aber ist konkret und positiv, eins mit der sittlichen Substanz des Staates. Beides läßt sich nicht umstandslos unter einen Hut bringen, nur selten sind die historischen Bedingungen einer glücklichen Liaison von Menschenrechten und Demokratie erfüllt. Noch heute argumentiert der Hegelianer und Carl Schmitt-Schüler Ernst Wolfgang Böckenförde ganz ähnlich und schlägt deshalb eine rigorose Entkoppelung der Menschenrechtspolitik von der Frage der Demokratie vor. ${ }^{6}$

Im Konflikt zwischen voluntas und ratio zieht die schwache Rechtsvernunft regelmäßig den kürzeren. Diese rechtsrealistische These teilt Hannah Arendt mit Carl Schmitt ebenso wie mit Karl Marx. ${ }^{7}$ Jeder Versuch, die zunächst nur moralischen Rechte des Menschen durch den souveränen Willen des Volkes zu verwirklichen, muß deshalb dazu führen, die Rechte des Individuums auf dem Altar des völkischen

2 H. Arendt (Fn. I), S. 371 , wo Arendt die eigentliche Aporte beschreibt: "Die gleichen Grundrechte wurden (in der Franzósischen Revolution - H. B.) einmal als das unverảußerliche Eigentum alles dessen, was Menschenantlitz trägt, erklart, um im selben Atemzug als die spezifisch nationalen Rechte eines souveranen Volkes, das diese Rechte seinem nationalen Befreiungskampf und seiner nationalen Geschichte verdankt, hingestellt zu werden. Die gleiche Nation stellte sich einerseits unter die Herrschaft des Gesetzes, dessen Quelle und Autoritat angeblich nur in den Menschenrechten zu suchen waren, und proklamierte doch gleichzeitig seine absolute Souveranıtat, demzufolge sie keinen allgemeinen, sondern nur nationalen Gesetzen unterworfen war und nichts Hoheres anerkannte als den souveränen Volkswillen, also sıch selbst. « Dadurch gelang es am Ende der Nation, »sich an die Stelle des Gesetzes « (Arendt (Fn. I), S. 370 ) zu setzen (vgl. ebd., S. 434,454 f.).

3 Die narzißtische Krankung ( Beleidigung ) der uneinholbar individuellen Selbstbestimmung durch das alle gleich behandelnde Gesetz ist einer der Ausgangspunkte für die Theorie der entzweiten Sittlichkeit ( Tragodie im Sittlichen «) beım jungen Hegel. Die Anerkennungslehre folgt dieser Spur, die sıch dann beim reifen Hegel in der Philosophie des Rechts verliert. Eine ähnliche Aporie der Rechte wie H. Arendt oder der junge Hegel konstruiert auch: J. Derrida, Gesetzeskraft, Frankfurt 1992.

4 H. Kelsen, Allgemeine Staatslehre, Berlin 1960, S. 34 I. Kelsen schreibt sogar mit Bezug auf die nur »volkerrechtsunmittelbare « Souveranıtat: Nur die Völkerrechtsordnung, keine staatlıche Rechtsordnung ist souveràn «. (S. $\left.33^{8}\right)$.

5 C.Schmitt, Verfassungslehre, Berlin 1928, S. 139 f., vgl. a. S. $22 \mathrm{ff.,} 83$ f., 107 f., $147,258 \mathrm{f}$

6 Vgl. E.-W. Bockenforde, "Das Unwahrscheinliche wollen. Demokratie, notwendige Forderung der Menschenrechte? «, in: FAZ vom 2. Mal 1996, S. N 6.

7 K. Marx, "Zur Judenfrage«, in: Marx/Engels, Werke Bd. 1, S. $347 \mathrm{ff}$. 
Verlangens zu opfern. Nur so - das ist Arendts Punkt - läßt sich die Aporie im Rahmen egalitärer Demokratie lösen. Die Konsequenz dieser Lösung ist fürchterlich: Terror und am Ende totalitäre Herrschaft.

Das liegt nicht nur am Widerstreben des zur Souveränität befreiten Volkes, sich selbst durch menschenrechtstaugliche Gesetze zu binden. Das totalitäre Ende liegt schon im ursprünglichen Begriff des Menschen, dem das Aufklärungszeitalter universelle Rechte zugeschrieben hatte, beschlossen. Denn dieser Begriff ist "weltlos", atomistisch und unpolitisch. ${ }^{8}$ Er ist - wie Billy Budd - zu gut für diese Welt. Dringt er von außen in die Pluralität einer konkreten politischen Tugendordnung ein, muß er sie und sich selbst zerstören: "Das Absolute, das (...) in dem Begriff der Menschenrechte zum Ausdruck kommt, muß ein Unheil werden, wenn es sich innerhalb des politischen Raums Geltung verschaffen will. « 9

Die 1789 verkündeten abstrakten Rechte eines maus allen Bindungen herausgelösten, völlig isolierten Wesens « konnten zu Bindung, Welt und Pluralität nur zurückfinden, indem man kurzerhand "den 'Menschen überhaupt identifizierte.$^{\circ}$ In der Französischen Revolution "vermengte sich ", schreibt Arendt, "die ganze Frage der Menschenrechte von vornherein mit der Frage nationaler Emanzipation und des Selbstbestimmungsrechts der Völker. Nur die emanzipierte Souveränität des Volkswillens, und zwar des Willens des eigenen Volkes, schien imstande, die Menschenrechte zu verwirklichen. Insofern die Französische Revolution die Menschheit als Familie von Nationen begriff, richtete sich der Begriff des Menschen, der den Menschenrechten zugrunde lag, nach dem Volk und nicht nach dem Individuum. " So gesehen gehören die Menschenrechte selbst zum Ursprung des Totalitarismus. Das ist nicht sonderlich überraschend, wenn man in Rechnung stellt, daß für Arendt der eigentliche Ursprung des Totalitarismus im neuzeitlichen Niedergang des Politischen als der res publica besteht. „Politikvergessenheit « könnte man Hannah Arendts These zum neuzeitlichen Weltbild nennen. Es ist ihre Version der Heideggerschen "Seins-Vergessenheit *. Arendts Wort für Politikvergessenheit ist "Weltlosigkeit".

"Weltlosigkeit « ist das Merkmal, das der Mensch der Menschenrechte mit dem Menschenmaterial totalitärer Herrschaft teilt. Die Erfindung eines weltlosen »Menschen überhaupt « setze, so Arendts erste These, den »Verlust der öffentlich gesicherten Gemeinschaft überhaupt«, den Verlust »der Fähigkeit zum Politischen « voraus. ${ }^{12}$ Aber erst in der Verbindung mit dem Demokratieprinzip - so die zweite These werde der latent totalitäre Charakter der Menschenrechte manifest.

Hannah Arendt kann sich die moderne, egalitäre Massendemokratie nur als homogenen Nationalstaat vorstellen. Wie Carl Schmitt setzt sie die Massendemokratie mit dem homogenen Volkskörper in eins. Das demokratische Staatsvolk moderner Gesellschaften ist in seiner großen Mehrzahl zu keiner überlegten Entscheidung und vernünftigen Diskussion im Stande. Vernünftig miteinander reden können immer nur die wenigen, die gebildet und mutig genug sind, sich für die allgemeinen Angelegenheiten einzusetzen und das persönliche Interesse hintanzustellen. Aber die Massen moderner Gesellschaften sind zutiefst konsumistisch und vor allem um ihr leibliches Wohl und ihren persönlichen Vorteil besorgt. Sie können nur, ohne zu überlegen, mit »ja« oder »nein« auf vorformulierte Fragen antworten und sind ge-

8 H. Arendt (Fn. 1), S. 454, 46 If.; vgl. a. H. Arendt, Über die Revolution, Munchen 1986, S. 36 ff., ssf., $237 \mathrm{f}$

9 H. Arendt, Über die Revolution (Fn. 8), S. 107.

$10 \mathrm{H}$. Arendt, Elemente und Ursprünge totaler Herrschaft (Fn. 1), S. 455 .

II H. Arendt (Fn. 1), S. 455

12 Ebd., S. $4^{63}$ 

sind vor allem durch präpolitische Bedürfnisse »des Bauches« bestimmt. Herrschaft degeneriert in der Massengesellschaft zwangsläufig zur - so Arendt - "Herrschaft des Niemand $\ll{ }^{13}$

\section{II}

Ich glaube, daß beide Thesen der Kritik nicht standhalten, weder die von der notwendigen "Weltlosigkeit« der Menschenrechte noch die von der tragischen Konstitution des modernen Nationalstaats. Schon historisch - und das ist mein erster Kritikpunkt - ist die Unterstellung, die Französische Revolution habe die Frage der Menschenrechte mit dem ethnisch verstandenen Selbstbestimmungsrecht der Völker vermengt, unzutreffend. Wie kürzlich noch einmal Rogers Brubaker in einer brillanten Untersuchung, die der Geschichte des Staatsbürgerrechts in Deutschland und Frankreich nachgeht, gezeigt hat, spielte das vorstaatliche "Selbstbestimmungsrecht der Völker « und die Herder entlehnte Vorstellung einer vor allem Recht existierenden "Familie der Nationen« weder in der Französischen Revolution noch im Selbstverständnis der späteren Republik eine nennenswerte Rolle. ${ }^{14}$ Erst nach dem Weltkrieg von 19 r $_{4}-1918$ ist auf Betreiben des amerikanischen Präsidenten Wilson das Selbstbestimmungsrecht der Völker, mit katastrophalen Folgen, in Europa zum Zuge gekommen, - und zwar vor allem in Mittel- und Osteuropa, nicht jedoch in Frankreich und im europäischen Westen. ${ }^{15}$ Anders als im ehemaligen Jugoslawien spielten ethnische Probleme auch im spanischen Bürgerkrieg in den zoer Jahren keine nennenswerte Rolle. Das Selbstverständnis der Französischen Republik war wie das der Revolution von 1789 assimilatorisch und juristisch bis hin zum Imperialismus, aber niemals ethnisch. Es ging der französischen Menschenrechtspolitik niemals um eine organische Familie von Völkern mit verschiedenen, geschichtlich geprägten Verfassungen, sondern eher um die geschichtsblinde Forderung, alle Menschen zu Franzosen zu machen und ihnen eine republikanische Verfassung französischen Typs aufzuprägen. Die These, die Nation habe den Staat erobert und das Recht zerstört, paßt auf Hitler und - setzt man »Partei« an die Stelle von »Nation «- auf Lenin und Stalin, nicht aber zur Französischen Revolution und zum Französischen Republikanismus. ${ }^{16}$

Hannah Arendt mißachtet in ihren Büchern über den Totalitarismus und über die Revolution durchgängig diesen fundamentalen Unterschied zwischen dem französischen und dem deutschen, zwischen dem juristischen und dem ethnischen Begriff des Volkes, zwischen der als Rechtsgenossenschaft konstitutierten Staats-Nation und der vorpolitischen und vorrechtlichen Kulturnation. Ihre These, im europäischen Nationalstaat liege der Ursprung des Totalitarismus verborgen, trifft deshalb nur auf den ethnisch definierten Nationalstaat zu. Nur dieser Staat tendiert dazu, die Nation an die Stelle des Rechtsstaats zu setzen und die moralischen Rechte des Individuums zu vernichten. Im juristisch konstituierten Nationalstaat des französi-

I3 Das ist in den 2oer Jahren die durchgangige Meinung unter den gebildeten Verachtern der Demokratie, sıe findet sich ebenso bei Robert Michels, Schumpeter oder Max Weber, der z. B. In Wirtschaft und Gesellschaft schreibt: „Demokratisierung und Demagogie gehoren zusammen.« (S. 861).

I4 R. Brubaker, Staats-Bürger, Hamburg 1994, vgl. a. meine Rezension: "Volkstaat oder Staatsvolk? « in: Dic Zeit $43 / 1994$, S. 76.

is Hannah Arendt geht statt dessen von der These aus, infolge des Versailler Vertrages sei es zu einer Verwestlichung des Ostens gekommen. Doch das Gegenteil scheint richtig zu sein. Nach Versailles ist das ethnische Verstandnis von Staatsburgerschaft ostlich des Rheins erst voll zum Zuge gekommen. Vgl. H. Arendt (Fn. I), S. 429 f.

r6 Zur These vgl. H. Arendt (Fn. 1), S. 370 f., 434, 446. 
schen Typs hingegen werden die moralischen Menschenrechte nicht vernichtet, sondern von der Moral getrennt und Zug um Zug in positives Recht verwandelt. Deshalb ist diese Form des Nationalstaats - anders als Arendt glaubt ${ }^{17}$ - mit einer republikanischen Verfassung nicht nur vereinbar, sie setzt sie voraus.

Die Positivierung der Menschenrechte zu einklagbarem Recht ist der entscheidende evolutionäre Fortschritt, der mit den Verfassungsrevolutionen des 18. Jahrhunderts begonnen hat. Dadurch wird zunächst die von Arendt befürchtete, gesinnungsethische Moralisierung des öffentlichen Raums verhindert. Sodann wird eine unbegrenzte, und insofern souveräne, demokratische Ausgestaltung der Freiheitsrechte des Einzelnen ermöglicht. Das hat in den westlichen Demokratien zu einer ebenso einleuchtenden wie historisch vergleichsweise erfolgreichen, wechselseitigen Stabilisierung von Menschenrechten und Volkssouveränität geführt. Die Positivierung ist entscheidend, aber das übersieht Arendt. Positives Recht kann nämlich nicht mehr aus der Moral oder der Vernunftnatur des Menschen abgeleitet werden und ist deshalb auf die freie Übereinstimmung aller Betroffenen als der einzigen noch verbliebenen Legitimationsquelle allen Rechts angewiesen. Schließlich bleibt auch das positivierte und damit zunächst territorial eingeschränkte Menschenrecht von der evolutionären Eigendynamik des Rechtssystems nicht unberührt. Diese Eigendynamik ausdifferenziertem Rechts tendiert von vornherein zu einer Weltrechtsordnung. Die Globalisierung des Rechts sprengt am Ende die nationalstaatlichen Grenzen und erzwingt Zug um Zug die völkerrechtliche Positivierung der Menschenrechre - auch wenn die internationalen Organisationen, die ihre entmoralisierte Durchsetzung sichern sollen, zunächst schwach bleiben. ${ }^{18}$ Das führt mich zu einer zweiten kritischen Bemerkung.

Hannah Arendt hat diesen Entwicklungsfortschritt, der ja durchaus ein "Fortschritt im Bewußtsein der Freiheit « ist, nicht wahrgenommen. Sie hat in den, nach Darwins Entdeckungen möglich gewordenen, Evolutionstheorien des 19. und 20. Jahrhunderts nur das Symptom eines Verfalls des Menschen, seine Rückbildung zu einer bloßen "Tiergattung " gesehen. ${ }^{19}$ Für sie war die Evolutionstheorie nur eine Variante totalitärer Geschichtsphilosophie. »Darwins Einführung des Begriffs der Entwicklung in die Natur, seine biologischen Konstruktionen, die alle darauf hinauslaufen, daß die Bewegung der Natur, nämlich ihre Entwicklung, nicht kreisförmig, sondern gradlinig verläuft in einer eindeutig angebbaren, fortschreitenden Richtung, besagt schließlich nichts anderes, als daß der moderne Geschichtsbegriff sich auch der Naturwissenschaften bemächtigt hat und daß der Bereich der Natur von dem Bereich des Geschichtlichen überwältigt wurde. ${ }^{20}$ Das Denken in revolutionären Stufen mußte ihr deshalb wie das »missing link « zwischen Französischer Revolution und Totalitarismus erscheinen.

Aber es gibt in der westlichen Welt auch eine republikanische Variante des Darwinismus. Wie nicht nur das Werk John Deweys zeigr, besteht in der Verbindung von Republikanismus und Evolution, von Demokratie und ziellosem Wachstum eine

I7 H. Arendt bestreitet wiederholt die Moglichkeit eınes republikanischen Nationalstaats. Vgl. z. B. Uber die Revolution (Fn. 8), 5.66, $215,222,318$ ff.

18 Vgl. H. Brunkhorst, "Paradigmenwechsel im Volkerrecht? Lehren aus Bosnien «, in: M. Lutz-Bachmann (Hg.), 200 Jahre $\bowtie$ Ewıger Frieden «?, Frankfurt 1996.

ig H. Arendt, Vıta actıva, S. 105, 21 s: „Es gibt (...) Gefahrensignale dafur, daß der Mensch sich anschicken konnte, sich in die Tiergattung zu verwandeln, von der er seit Darwin abzustammen meint. «

$20 \mathrm{Vgl}$. z. B. Elemente und Ursprunge (Fn. 1), S. 709. Daß dem nicht so ist, zcigt D. Dennett, Darwins Dangerous Idea. Evolution and the Meanings of Life, London 1993; vgl. a. D. Dennett, "Es geht auch ohne Gott und Geist«, in: DIE ZEIT 8, 16. Febr. 1996, S. 30-31; zur wichtigen Unterscheidung von Evolution und Geschichte vgl. a. J. Habermas, Zur Rekonstruktion des Historischen Materialısmus, Frankfurt 1976, S. $200 \mathrm{ff}$. und N. Luhmann, Soziologische Aufklarung, Bd. 2, Opladen ig7s, S. i soff., $193 \mathrm{ff}$. 
antitotalitäre Alternative zu der von Arendt zutreffend beobachteten und eindrucksvoll beschriebenen »Bewegungssüchtigkeit totalitärer Bewegungen «. ${ }^{21}$ John Dewey könnte an dieser Stelle fragen: Was ist eigentlich falsch an unbegrenztem Fortschritt und an der Auflösung starrer Strukturen in fließende und unvorsehbare Bewegung? Was ist falsch an der Utopie einer unvorstellbar besseren Zukunft, wenn diese Utopie nicht mit Totalitarismus, sondern mit einem egalitären, demokratischen Verfassungsstaat verbunden ist?

Hannah Arendt hat stets großen Wert auf die Ideen eines neuen Anfangs und einer neuen Gründung der republikanischen Ordnung gelegt. Politik war für sie immer zuallererst ein gemeinsames kooperatives Projekt, in dem Herkunft, ererbtes Privileg und Tradition nicht zählen. Aber sie hat es entschieden abgelehnt, den neuen Anfang eines Lebens oder eines politischen Projekts Begriffen von Evolution und Fortschritt unterzuordnen. Die neue politische Ordnung stellt einen radikalen Bruch mit der Vergangenheit dar, ist ein vollkommen diskontinuierliches Ereignis, niemals Teil eines geordneten Entwicklungsganges, dessen relative Vernunft nachträglich rekonstruiert und eingesehen werden könnte. Deweys Ideen einer Evolution ohne Telos und einer Utopie unbegrenzten Wachstums waren für sie ebenso totalitär wie diejenigen aus Stalins "Diamat«. Aber das ist falsch. Es gibt innerhalb der Moderne eine ganze Reihe utopischer Projekte, die sich sehr voneinander unterscheiden. Einige funktionieren, andere nicht. Einige sind totalitär, andere demokratisch und liberal. Die Moderne scheint dazu verdammt, sie alle ausprobieren zu müssen. Experimentalismus ist ihr Schicksal, aber auch ihre große Chance. Wenn wir Glück haben, gelingt es uns, die besten Projekte festzuhalten, sie zu verbessern und durch bessere zu ersetzen.

Vom evolutionistischen Standpunkt aus betrachtet, ist nichts besonders Gefährliches oder Falsches an der Vorstellung, das moderne Entwicklungsstadium autonomer Subjektivität und republikanischer Menschenrechtspolitik könne eines Tages einem höheren Niveau sozialer Integration weichen, auf dem wir mit den Problemen, mit denen wir uns heute herumschlagen, besser fertig werden. Warum sollten wir nicht eines Tages zu sehr viel egalitäreren Gesellschaften als wir sie uns heute vorstellen können, kommen? Gesellschaften, die neue und differenzierte Arten der Freiheit entdecken und die nicht länger politisch integriert sind, weder im aristotelischen noch im rousseauschen Sinne des Wortes. So großartig war »das Politische« auch nicht, und seine Problemlösungskapazität ist offensichtlich geringer als die der Wirtschaft oder der Wissenschaft.

Weil Hannah Arendt die Evolutionstheorie ablehnt, besteht der einzige Ausweg, der ihr bleibt, im Rückzug aus der Geschichte des okzidentalen Rationalismus, in der Rückwendung zum Ursprung des Politischen in Rom und Athen und in der Rehabilitierung der teleologischen Bestimmung des Menschen als eines politischen Lebewesens. $^{22}$ Dieses Wesen ist eine Perfektionsgestalt des Menschen, die keiner Entwicklung unterliegt und sich deshalb auch nicht ändern kann. Wir können ihr jederzeit und überall immer nur mehr oder weniger nahe kommen. Und immer gibt es Menschen, die ihr nahe kommen und solche, die das nicht schaffen. Erstere sind im emphatischen Sinn Bürger, die sich erfolgreich für das Gemeinwohl engagieren, letztere »bloße« Menschen, in der Antike waren das die Sklaven, die Frauen und die Barbaren. Wo Autoren wie Dewey ein entwicklungsgeschichtliches Kontinuum sehen, sieht Arendt einen trennenden Abgrund, der zwischen Tieren und Menschen,

21 Elemente und Ursprunge (Fn. 1), S. 495 , vgl. a. S. $412,528,610$ ff, 708.

22 Den Ruckzug aus der Geschichte telt sie mit anderen Heideggerschulern. Vgl. z. B. K. Lowith, Weltgeschichte und Heilsgeschehen, Stuttgart 1990. H. Marcuse, Truebstruktur und Gesellschaft, Frankfurt 1965 . 
Menschen und Bürgern, politischen und unpolitischen Lebewesen, öffentlichem und privatem Dasein klafft. Wir sollten, denke ich, diesen metaphysischen Idealismus und sein großes Narrativ vom "politischen Lebewesen" vergessen und statt dessen John Deweys egalitären Antiaristotelismus akzeptieren, daß »der Mensch ebenso sehr ein konsumierendes und sportbegeistertes Tier ist wie ein politisches. ${ }^{22 a}$ Und wir sollten ergänzen: Es mag ja sein, daß der Mensch in einer fernen Vergangenheit in bestimmten Regionen der Welt ein politisches Tier war, aber in unseren Zeiten ist er das schon lange nicht mehr.

Meines Erachtens besteht die Stärke und Aktualität von Hannah Arendts Büchern in der Erinnerung an die zentrale Rolle des Streits der Meinungen und des öffentlichen Diskurses in einer modernen Republik. Wenn sie im »Recht, Rechte zu haben « das einzige Menschenrecht sieht und unter Rechten nicht primär die subjektiven $\mathrm{Ab}-$ wehrrechte eines "völlig isolierten Wesens « versteht, sondern intersubjekte Rechte, die die Bürger sich - wie schon Kant gesehen hatte ${ }^{23}$ - zunächst einmal wechselseitig einräumen müssen, dann hat sie einen wichtigen Schritt zur Überwindung des liberalen Atomismus und des abstrakten Naturrechts, an dem noch Kant festgehalten hatte, getan. ${ }^{24}$ Kein Recht und auch kein Menschenrecht darf außerhalb oder oberhalb des öffentlichen Lebens in einer Natur verankert werden, die jeder Debatte entzogen ist. Weder unsere Rechte noch der Wille des Volkes ist etwas, was irgendwo außerhalb des öffentlichen Raums existiert und deshalb in diesem nicht zur Debatte stehen könnte. Soweit folge ich Arendt.

Aber - und damit komme ich zu meinem letzten kritischen Punkt - sie geht viel zu weit hinter die Moderne zurück, wenn sie die zentrale Bedeutung des öffentlichen Diskurses für eine Republik mit dem Vorrang des öffentlichen gegenüber dem privaten Leben, der politischen gegenüber der unpolitischen Existenz, der Würde des Bürgers gegenüber der Würde des bloßen Menschen, der Bürgerrechte gegenüber den Menschenrechten, verwechselt. Deshalb assimiliert sie auch das "Recht, Rechte zu haben «, sofort an den Nomos einer sittlichen Tugendgemeinschaft. ${ }^{25}$ Das aber ist mit dem modernen, positiven Recht, das Arendt mit guten Gründen gegen den Totalitarismus verteidigt, nicht verträglich. ${ }^{26}$

Arendt gelingt es nicht, die Aporie mit der Formel vom Recht auf konkrete Bürgerrechte bzw. positive Freiheitsrechte aufzulösen. Denn entweder ist das Recht auf solche Rechte selbst ebenso abstrakt wie die Menschenrechte des 18. Jahrhunderts, dann verschiebt sich das Problem von einem Metarecht zum nächsten. Oder das Recht auf jene Rechte ist selbst konkret und substantiell, dann widerspricht es sich selbst, weil es dann - frei nach Russell - selbst zur Menge der Rechte gehört, die es als Rechte allererst spezifizieren soll.

22a J. Dewey, "The Public and its Problems «, Later Works 2, Carbondale 1984, S. 23 menne Ubers.

23 I. Kant, Metaphysik der Sitten, Werke VIII, Frankfurt 1977, S. 345 (AB 45).

24 Vgl. Elemente und Ursprunge (Fn. I), S. ${ }_{4} 62,468 \mathrm{f}$.

25 Arendt (Fn. 1) S. $462 \mathrm{ff}$. Vgl. a. dies., "Es gibt nur ein einzıges Menschenrecht", in: Die Wandlung, Dezember 1949, S. 754-770. (Der Text ist weitgehend identısch mit "Elemente und Ursprunge* (Fn. I), S. 452 ff.) "Das Recht, Rechte zu haben «, schreibt sie, "1st gleichbedeutend damit, in einem Bezıehungssystem zu leben, in dem man aufgrund von Handlungen und Meinungen beurteilt wird. (Elemente und Ursprunge (Fn. y), S. 462). Diese Vorstellung von Recht und Rechten ist eher dem »konkreten Ordnungsdenken « Carl Schmitts verwandt als dem rechtspositivistischen Formalismus, der seit Hobbes zur Sprache der Menschenrechte geworden ist. (Vgl. C. Schmitt, Über die dreı Arten des rechtswissenschaftlıchen Denkens, Hamburg 1934). Es gibt ubrıgens beı Hobbes zwei wichtıge Menschenrechte, die im posituvistischen Gesetzesbegriff implizit enthalten sind: das Recht, wegzulaufen und sich dem Zugriff des Politischen auf das eigene Leben zu entzıehen; und das Recht, zu denken, was man will. Arendts Kritik an Hobbes ist sehr einseitıg und blınd fur dessen liberalen Kerngedanken. (Vgl. Elemente und Ursprunge (Fn. r), S. 243 f.: Hobbes ist keın Apologet der Tyrannis, wie Arendt behauptet, sondern ein Theoretiker des Rechtsstaats.)

26 Vgl. Arendt (Fn. I), S. 706 ff. 
Es gibt freilich einen gewissen Fortschritt in Arendts eigenem Werk. Zwischen »Vita

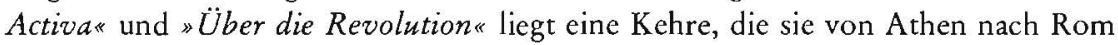
und zur römischen Idee der Gesetzesherrschaft führt. ${ }^{26 a}$ Aber auch auf römischem Boden bleibt man zunächst klassischer Republikaner, fern von allen - erst jüdischchristlichen und dann aufgeklärt modernen - Emanzipationsidealen. Auch der Begriff des positiven Rechts ist - entgegen Hegels voreiliger Unterstellung - römischem Denken fremd. Das erkennt man unschwer an der zentralen Rolle des Zensors, des zwangsbefugten Sittenwächters im antiken Rom. Diese Rolle, die das Recht in den Nomos einer konkreten Tugendordnung einbindet, ist gleich weit von der Amerikanischen wie von der Französischen Revolution entfernt.

Während der klassische Republikanismus stets von der Gleichheit der Bürger und der Ungleichheit der Menschen ausging, beerbt der moderne Republikanismus von Locke über Rousseau bis Kant und Dewey die biblische Vorstellung von der Gleichheit aller Menschen. An die Stelle des Dualismus zwischen dem unpolitischen „Verlangen nach Befreiung « und dem politischen »Willen zur Freiheit « tritt ein Kontinuum. ${ }^{27}$ Der Dualismus, den Arendt mit Aristoteles teilt, ist blind für das entwicklungsgeschichtlich Neue der modernen Massendemokratie: die Integration von Menschen- und Bürgerrechten. In ihr drückt sich nichts anderes aus, als das Projekt einer Versöhnung der republikanischen Kritik an jeder Herrschaft von Bürgern über Bürger mit der emanzipatorischen Kritik an jeder Herrschaft von Menschen über Menschen.

In einem Essay über »Religion und Politik « bezieht sich Arendt auf Lincolns »Gettysburg Adress $\ll .{ }^{28}$ Sie zitiert die berühmte Definition der Demokratie als "government of the people, by the people, for the people «, und sie betrachtet es als die schlagende Wahrheit des Textes, daß die modernen Amerikaner "politisch nicht länger in einer religiösen Welt leben « würden. ${ }^{29} \mathrm{Zu}$ Recht behauptet sie, daß die Idee der politischen Selbstorganisation eines Volkes weder christliche noch jüdische noch in sonst einem monotheistischen Verständnis religiöse Wurzeln hätte. Der Ursprung des Politischen ist zweifellos die heidnische Welt Athens und Roms.

Aber die ausschließlich republikanische Lektüre von Lincolns epochaler Gefallenenrede ist eine ebenso bewußte wie gezielte Fehllektüre. Gleich zu Beginn hebt Lincoln nämlich hervor, »that all men are created equal «, und das hat damals jeder direkt auf die Emanzipation der Sklaven als Menschen (und nicht primär als Bürger) bezogen. Perikles wäre in seiner früheren, nicht minder epochalen Gefallenenrede niemals so töricht gewesen, die Idee politischer Freiheit mit Sklavenemanzipation und »equal creation « in Verbindung zu bringen. Die Botschaft solcher Emanzipation war den Griechen, wie Paulus wußte, eben »eine Torheit«.

Perikles hätte ebensowenig, wie Lincoln es getan hat, die »Polis « und ihre Verfassung mit einem globalen demokratischen Projekt, einem "unfinished work «, das niemals "shall perish from the earth «, in Verbindung gebracht. ${ }^{30}$ Was für Perikles allein zählte, war die Zukunft seiner Stadt, niemals die Zukunft der Demokratie als universelles Menschheitsprojekt. Für Perikles wäre so ein Projekt nichts anderes als eine unpolitische Utopie von Idioten gewesen. Für Lincoln aber ist es »the great task

26a Darauf hat Andrew Arato in einem noch unpublizierten Vortrag (auf der Arendt-Konferenz in Cambridge/Mass. in diesem Frubjahr) aufmerksam gemacht.

27 Zum Dualismus vgl. Uber die Revolution (Fn. 8), S. 39.

$28 \mathrm{H}$. Arendt, Zwischen Vergangenheıt und Zukunft. Übungen im polıtischen Denken I, Munchen 1994 , S. 305-326; A. Lincoln, "Adress Delivered at the Dedication of the Cemetry at Gettysburg ", Nov. I9, 1863, in: A. Lincoln, His Speeches and Writings, New York 1969, S.734; vgl. a. G. Wills, Lincoln at Gettysburg. The Words that Remade America, New York 1992.

29 H. Arendt (Fn. 28), S. 310 E.

30 A. Lincoln (Fn. 28), S. 734 . 
remaining before us «. ${ }^{33}$ Für ihn sind - anders als für Arendt, Carl Schmitt oder auch Böckenförde - Bürgerdemokratie und Menschheitsdemokratie ein und dasselbe Projekt. Die politische Pointe der 272 Worte Lincolns nach der grauenvollen Schlacht bei Gettysburg besteht allein darin, daß es keinen Begriff moderner Demokratie gibt, der nicht innerlich mit der Idee der Gleichheit und Emanzipation aller Männer und Frauen verbunden wäre. Es gibt deshalb keine moderne Demokratie ohne das monotheistische Erbe der jüdisch-christlichen Idee universeller Solidarität.

\section{Ulrich Mückenberger Kritik des »Arbeitsrechtlichen Beschäftigungsförderungsgesetzes «*}

\section{Der arbeitsmarkt- und gesellschaftspolitische Kontext}

Das heute zur Erörterung anstehende Gesetz darf nicht als vereinzelte arbeitsrechtliche Maßnahme betrachtet und bewertet werden. Es steht vielmehr im Kontext zweier arbeitsmarktpolitischen Entwicklungen von geradezu historischer Bedeutung:

- Einmal hat die Massenarbeitslosigkeit in der Bundesrepublik mit weit über 4 Millionen Arbeitslosen einen Stand erreicht, der in der Nachkriegsgeschichte einmalig ist. Die Dramatik, die mit Massenarbeitslosigkeit verbunden ist - soziale Ausgrenzung, Armut, Kriminalität, Fremdenfeindlichkeit usw. -, drängt sich jedem, der diese Gesellschaft offenen Auges betrachtet, auf. Sie verlangt im wahrsten Sinne radikale Lernprozesse, die Fähigkeit und Bereitschaft aller mit dem Arbeitsmarkt beschäftigten gesellschaftlichen Akteure zur Umsteuerung. Manche sprechen in diesem Zusammenhang von der Notwendigkeit eines »neuen Gesellschaftsvertrages«.

- Zum anderen hat es aber Ende letzten, Anfang dieses Jahres den Ansatz zu einer solchen radikalen Umsteuerung gegeben, den bis dahin niemand für möglich gehalten hätte: Die auf die IG Metall zurückgehende Initiative »Bündnis für Arbeit«. Die IG Metall hatte, ohne ihre Auffassung, daß die Lohnhöhe nicht die entscheidende Variable für die Arbeitsplatzzahl sei, aufzugeben, Lohnzurückhaltung im Gegenzuge zur garantierten Arbeitsplatzschaffung durch die Mitglieder der Arbeitgeberverbände angeboten. Das Hintergrundereignis dieses Vorschlages war das in der deutschen Nachkriegsgeschichte umwälzende »VW-Modell«. Dort war auf Initiative des Arbeitsdirektors hin ein Abkommen zwischen Unternehmensleitung, Gewerkschaft und Betriebsrat zustande gekommen, das drohende Massenentlassungen durch einschneidende Arbeitszeitverkürzungen mit nur teilweisem Lohnausgleich vermied. »Radikal« war dieses Modell deshalb, weil beide beteiligte Seiten dabei bislang fest behauptete Tabus preisgaben: Die Arbeitgeberseite, daß Arbeitszeitver-

* Geringfugig bearbeiteter Text einer Stellungnahme zur Vorberettung der offenthichen Anhorung des Ausschusses fur Arbeit und Sozıalordnung des Deutschen Bundestags am 10. Juni 1996 in Bonn (AusschußDrucksache 13/63b). Grundlage der Ausschuß-Anhorung war der Gesetzentwurf BT-Drs. 13/1412. Er wurde in der aufgrund von Beschlußempfehlung und Bericht des Ausschusses fur Arbeit und Sozialordnung vom 26. Juni (Drs. 13/5107) modifizıerten Form am 28. Jun1 1996 vom Deutschen Bundestag angenommen (BR-Drs. $461 / 96)$.
} 